\title{
Interactions of a counter-rotating vortex pair at multiple offsets
}

\author{
Kyle J. Forster ${ }^{\mathrm{a}, *}$, Tracie J. Barber ${ }^{\mathrm{a}}$, Sammy Diasinos ${ }^{\mathrm{b}}$, Graham Doig ${ }^{\mathrm{c}, \mathrm{a}}$ \\ a School of Mechanical and Manufacturing Engineering UNSW Australia, NSW 2052, Australia \\ ${ }^{\mathrm{b}}$ Department of Engineering, Macquarie University, North Ryde, NSW 2109, Australia \\ ${ }^{\text {c } A e r o s p a c e ~ E n g i n e e r i n g ~ D e p a r t m e n t, ~ C a l i f o r n i a ~ P o l y t e c h n i c ~ S t a t e ~ U n i v e r s i t y, ~ C A ~ 93407, ~ U S A ~}$
}

\section{A R T I C L E I N F O}

Keywords:

Aerodynamics

Particle Image Velocimetry

Vortex interaction

Streamwise vortices

Counter-rotating vortices

Vortex instability

\begin{abstract}
A B S T R A C T
The interactions between two streamwise vortices were investigated by wind tunnel testing of two NACA0012 vanes at various lateral offsets. One vane was spaced 10 chord lengths (C) downstream of the other, with both at an angle of incidence of 8 degrees and a Reynolds number of $7 \quad 10^{4}$. The evolution of the vortex pair was observed until 6.5C behind the downstream vane using Particle Image Velocimetry (PIV). It was found that proximity of the upstream vortex to the downstream vane had a significant effect on the rotational rate of the subsequent vortex pair, with far offset cases having little rotation, and near field cases having angle changes of 19.6 degrees per chord length travelled downstream. At the point of vortex impingement on the downstream vane, the rotational rate dropped to near zero due to a significant strength reduction of both vortices. The point of strongest interaction was found to be laterally offset from the point of closest vortex proximity to the downstream vane by $0.15 \mathrm{C}$, with the vortex on the suction side of the vane. In the offset range investigated, a significant instability was observed in only the upstream vortex. These instabilities increased as the proximity between the vortices decreased, peaking where the vortex interaction was strongest.
\end{abstract}

\section{Introduction}

Vortex generators operating in boundary layers, turbomachinery blade interactions, wind turbines and aircraft flying in formation can all produce vortex interactions with multiple streamwise vortices in close proximity to each other [1-6]. Streamwise vortex/structure interactions have been studied considerably less than either parallel or normal vortex/structure interactions [7], particularly relating to the effects of the upstream vortex migration. Vortices of a vortex pair have been typically deployed from the same streamwise location, limiting their proximity. However, close interactions are important conditions to understand in order to provide a knowledge base for practical vortex applications, where upstream vortices may move in locations on either side of a vortex producing obstacle, such as a wing or vane.

Interacting pairs of streamwise vortices can be classified into either counter-rotating or co-rotating configurations. Counterrotating pairs exhibit a number of instabilities when placed in close proximity to one another, including long wavelength (Crow [8]), short wavelength (elliptic [9]) and spiral $[10,11]$. The Crow instability is described through a solution to a linear wave system,

\footnotetext{
* Corresponding author.

E-mail address: kyle@forsters.com.au (K.J. Forster).
}

which describes the deviations of counter-rotating vortex pairs [8]. Once the vortex cores reach a certain proximity or cutoff distance the two wakes unify into vortex rings and rapidly breakdown. Vortices that break down or dissipate in short distances and timeframes do not have a long enough duration for waves to form, and as such are not subject to the Crow instability. Using these models, it has been found that all counter-rotating pairs are inherently unstable regarding the long wave Crow instability [12-14]. For vortices of unequal strength, the Crow instability can manifest itself at much shorter wavelengths than for an equal strength case. This has been simulated numerically using Computational Fluid Dynamics (CFD), and it has been found that a medium length instability is present where the weaker vortex is drawn around the primary vortex [15].

The short wave (elliptic) instability is identified in counter and co-rotating pairs by a streamtube in the core of the vortex with a diameter approximately half that of the instabilities wavelength. This instability is caused fundamentally by a resonance of two Kelvin waves (a sinusoidal deformation) within the vortex core as driven by the strain field induced by the other vortex [16]. Like the Crow instability, it is modified by differing axial velocity components and vortex strengths. The effect of these instabilities on migration and core size in practical upstream/downstream vortex layouts is currently unknown. 


\section{Nomenclature}

$\begin{array}{ll}R_{0.1} & \text { average radius of vortex at } 0.1 \text { vorticity threshold } \\ A_{0.1} & \text { total area of vortex at } 0.1 \text { vorticity threshold } \\ \Gamma & \text { circulation } \\ X_{c} & X \text { core location } \\ Y_{c} & Y \text { core location }\end{array}$

$C \quad$ chord length

$R_{e} \quad$ Reynolds number, based off chord length

For free flow (unbounded) inviscid cases any vortex pair will maintain a constant core separation distance due to the conservation of angular momentum [9]. For a symmetric (equal circulation), counter-rotating case, this will mean that the pair will translate along the vortex pair centre axis, while for a case with unequal circulations there will be an orbital motion [9]. These migrations have also been observed in water tunnel testing [17], where dye marker injected into the cores of a pair of counter-rotating vortices showed a near linear trend in downwards motion of an equal strength pair. This motion increases in magnitude as vortex swirl is increased through varying the angle of attack of the vortex generation blades.

The interactions of a streamwise vortex with a wingtip at close range have also been computationally investigated $[7,11]$. By aligning an incident vortex with the tip of a downstream vane, the energy of the vortex system is increased in the near range, however more rapid energy attenuation occurs downstream. When the vortex is positioned inboard of the tip, it reduces the tip vortex size and strength, while placing it outboard of the wingtip enhances the wingtip vortex [7]. Reducing the distance of the incident vortex to the wingtip has been found to increase the magnitude of the turbulence production from the resultant vortex interaction [11]. It has experimentally been found that a counter-rotating wing configuration with a $2.5 \mathrm{C}$ streamwise wing spacing can substantially improve rear wing L/D by up to $24 \%$ at an overlap of $5 \%$ of the wingspan [18]. Such a configuration causes migration of the rear vortex towards the root of the rear wing, however the downstream consequences of these interactions have not been characterised for more than one chord length downstream. These effects have also not been evaluated at different vortex distances from the suction and pressure sides of the downstream vane.

Adverse pressure gradients produced by downstream geometries can interact with and disrupt the path of an existing vortex. A significant obstruction in the path of a vortex will cause the vortex to transition into either a spiral or bubble breakdown mode [19]. This vortex breakdown location is dependent on the swirl number (controlled by the angle of incidence of the upstream vane) and the adverse pressure gradient. If the adverse pressure gradient is not sufficient to cause breakdown, only slow diffusion of the core through viscous mechanisms will occur.

Due to the swirling nature of vortices, they act as pressure gradient amplifiers in the sense that an induced pressure gradient in the freestream will be substantially increased at the vortex core [20]. A probe placed near a vortex causes substantial upstream migration of the breakdown location [21]. As such, either Laser Doppler Anemometry (LDA) or Particle Image Velocimetry (PIV) must be used for accurate experimental results for steady vortices. However averaging point measurements can result in errors of up to $35 \%$ in tangential velocity in meandering vortex cases, emphasising the importance of a global measurement technique for meandering or unstable vortex analysis [22,23].

The work described in this paper investigates the near field interactions of a vortex produced by an upstream vortex with a downstream vane. PIV analyses have been performed for a wide variety of vane offsets at multiple downstream locations, allowing inspection of both the paths of the vortices and the meandering of the vortex pairs. Characterisation of near-field counter-rotating vortex interactions has been achieved, and the effects of generating a vortex in a flow field with a pre-existing vortex structure are found.

\section{Experimental setup}

The present study considers the interaction of two streamwise vortices produced by two NACA 0012 vanes. One vane was located 10 chord lengths $(C)$ downstream of the other, as can be seen in Fig. 1. This configuration was chosen as it allows interactions between vortices to occur at extremely close proximities that cannot be observed if the vortices are deployed at the same locations. This is also representative of the effects of a pre-existing vortex in a flow interacting with a vortex producing device. An angle of attack of 8 degrees on each vane has been used for all cases, with a square-edged tip. Higher angles of attack decreased the vortex stability, with unsteady breakdown becoming observable for a single vortex case at 12 degrees. Multiple offsets were tested from $0.6 \mathrm{C}$ to $0.5 \mathrm{C}$ in increments of $0.1 \mathrm{C}$, with a finer spacing of $0.05 \mathrm{C}$ between $0.4 \mathrm{C}$ and $\mathrm{OC}$.

The $x$-axis is in the direction of the flow, with positive downstream, the $Y$ axis is across the tunnel and the $Z$ axis is in the vertical direction. As such, the rear vane quarter chord was located at $X=10$ C, with the vane root at $Z=1.5 \mathrm{C}$.

Planar slices of the flowfield were captured using PIV at $0.5 \mathrm{C}$ intervals from $1.5 \mathrm{C}$ back from the quarter chord of the trailing vane to $7 \mathrm{C}$ back. These correspond to $11.5 \mathrm{C}$ and $17 \mathrm{C}$ from the leading vane respectively. The laser sheet was not moved closer than $11.5 \mathrm{C}$ as the reflections from the vanes began to distort the results. The experiment was performed at a Reynolds number of approximately $710^{4}$ based on chord length. At $7 \quad 10^{4}$ the vortex shedding from a NACA0012 airfoil at 8 degrees angle of attack is within the supercritical region [24] and therefore any Reynolds number lower than $6 \quad 10^{4}$ at this angle of attack will result in a shedding regime that is not indicative of higher Reynolds number scenarios. Running the tunnel as slow as possible within the acceptable Reynolds number range minimised vibration of the diffuser expansion, camera mounting and test section caused by the operation of the fan, thus minimising imaging errors.

\subsection{Wind tunnel}

Experiments were performed in the Macquarie University open return, closed section wind tunnel. This tunnel has a 610610 $\mathrm{mm}$ (24 24 in.) octagonal test section with a $1900 \mathrm{~mm}\left(6^{\prime} 3^{\prime \prime}\right)$ length. Optical access is through a glass window on the top of the test section and removable windows on the side. The test section was characterised using a Turbulent Flow Instrumentation 100 Series Cobra probe, giving a peak turbulence intensity of $0.35 \%$ and average of $0.25 \%$. Velocity uniformity was measured as better than $1 \%$ variance, and flow angularity was found to vary by 1 degree across the test section inlet. The wind tunnel speed was electronically controlled through a National Instruments MyRIO, with the pressure sensors calibrated against a temperature 


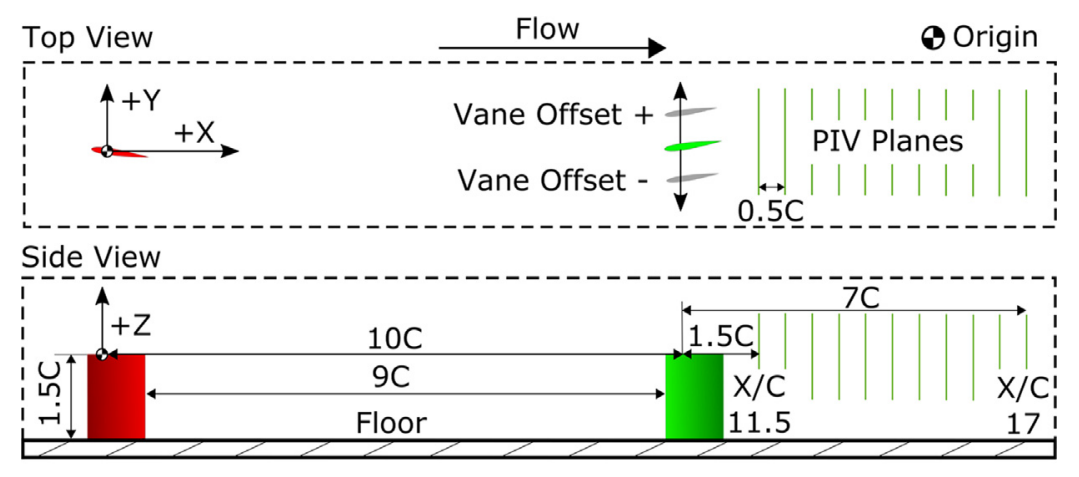

Fig. 1. Vane layout diagram, origin is at quarter chord tip of front vane.

controlled Baratron 120AD Differential Capacitance Manometer. Streamwise velocity variance was held to within $0.38 \%$.

A separate elevated ground is mounted to the floor of the tunnel with a rounded front splitter to minimise the effects of the preexisting layer in the test section. This ground is mounted $100 \mathrm{~mm}$ above the tunnel floor on two steel rails. To reduce the influence of secondary structures resulting from horseshoe vortices or boundary layer stripping, the vanes were sized to be significantly taller than the boundary layer. This prevents strong interactions with these secondary structures, allowing the study to focus on the interactions of the two tip vortices. The vanes have a chord of $80 \mathrm{~mm}$ and a span of $120 \mathrm{~mm}$, and are painted matte black to minimise reflections.The boundary layer at the location of the rear vane was experimentally measured to be $5 \mathrm{~mm}$ thick at $80 \%$ of the freestream velocity and $20 \mathrm{~mm}$ thick at $95 \%$ of the freestream velocity. A schematic of this setup can be seen in Fig. 2 .

\subsection{PIV setup}

A planar two component PIV system was used to capture the vortex dynamics. Due to the large expansion length of the Macquarie University wind tunnel, the camera was placed inside the expansion itself rather than using a mirror system. This allowed the camera to be positioned $2100 \mathrm{~mm}$ downstream of the test section and $2380 \mathrm{~mm}$ to the nearest image plane, giving a maximum perspective bias of 1.6 degrees per side on a $133 \mathrm{~mm}$ wide observation plane with a $120 \mathrm{~mm}$ lens. Planar PIV can produce projection errors when the out of plane motion is dominant [25]. However, this can be substantially reduced by lowering the per- spective error from the camera, reducing the motion to as close to the in-plane component as possible. For the comparison setup 2D and stereoscopic PIV of Yoon and Lee [25], it was found that a camera with an effective perspective angle of 5.71 degrees per side could produce an absolute maximum error of $20.8 \%$ in instantaneous in-plane velocity where the out-of-plane component was proportionally large in a vortex driven flow. By reducing this angle to 1.6 degrees through placing the camera much further away and using a zoom lens, as per the setup described in this paper, the maximum projection error is reduced to $5.8 \%$ under the same conditions. It should be noted that this error is at the edges of the observation window, and is not indicative of the errors near the centre, which will approach zero projection error as the centre is reached. The resultant vorticity field is consequently less affected due to the steepness of the velocity gradients in the core of the vortex as opposed to the shallow gradient of projection error induced velocities. By superimposing the calculated projection error of a uniform streamwise velocity field on the captured time-resolved PIV data, the error in peak vortex core velocity was found to be below $4 \%$ against the absolute velocity field, with an imperceptible change in the vorticity field. This resulted in a negligible change in the calculated core location and circulation. Focus was controlled remotely. By placing the camera this far downstream of the test section, there was no observable difference to the flow in characterisation measurements obtained through the tunnel section. The expansion section of the tunnel was on isolated mounts from the tunnel fan, minimising vibration. Over a test of 200 image pairs, the tip of the rear vane was found to have a maximum displacement change of 1 pixel during the entire sampling time.

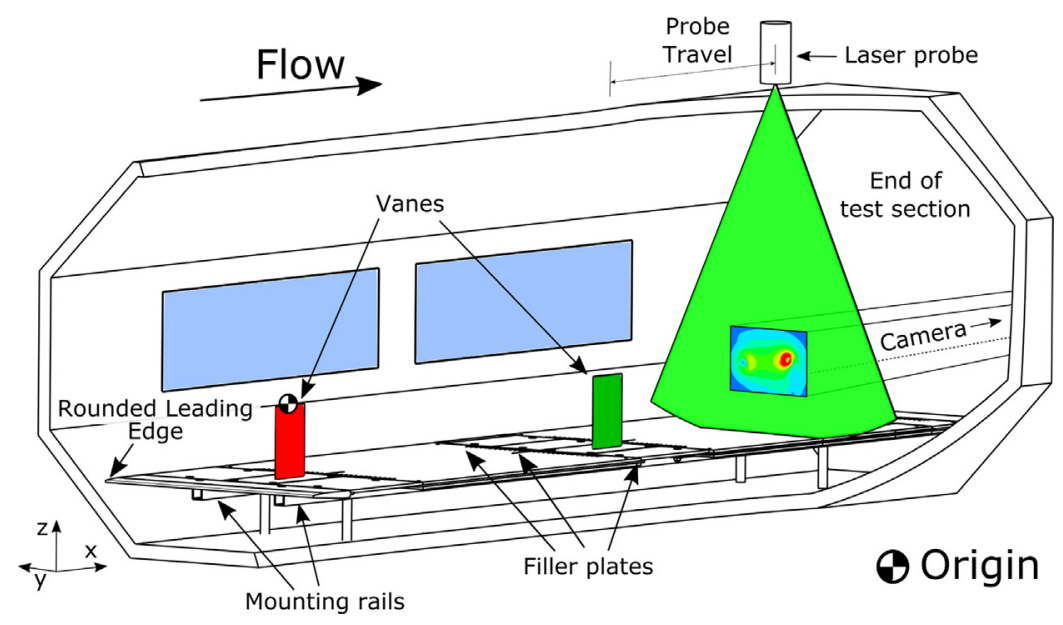

Fig. 2. Cutaway diagram of tunnel test section. 
Tracking of camera vibrations between images of an image pair was performed through a Gaussian fit tracking of the illuminated wingtip while the tunnel was running. This yielded a vibrational displacement maximum of $0.0471 \mathrm{px}$ between the two images of a pair, which is within the margin of error of Gaussian subpixel tracking of just below $0.1 \mathrm{px}$ at low signal to noise ratio as identified by Saunter [26].

Laser access to the tunnel was through a glass window in the top of the test section. The laser beam was sent to this location via a periscope connected to a Dantec 3-axis computer controlled traverse. This traverse was restricted to only allow laser sheet movement along the axis of the tunnel. The laser used was a dual-cavity Nd:YAG laser (Quantel EverGreen) with an output of $200 \mathrm{~mJ}$ per pulse at $532 \mathrm{~nm}$ wavelength and a repetition rate of $15 \mathrm{~Hz}$. Synchronisation between laser and camera was performed with an ILA synchroniser. Laser pulses were delivered at $55 \mu \mathrm{s}$ apart as any higher resulted in significant out of plane migration of particles. This is equivalent to a downstream movement of $0.665 \mathrm{~mm}$ per particle at the freestream velocity. The laser sheet thickness varies throughout the observation window as a result of the focus, with an average thickness of approximately $4 \mathrm{~mm}$ through the region of interest. This large thickness was selected to minimise the amount of out-of-plane pair loss [27], with the laser being run at maximum power to compensate for the reduced sheet intensity. By combining this thickness with the short pulse separation of $55 \mu$ s and a high particle seeding density the effective number of particle image pairs in the interrogation window was kept above 10 , giving a greater than $98 \%$ valid detection probability [27], thus being sufficient to compensate for the predominately out of plane flow component. Validation of post-processed data was performed by excluding points with vorticity gradients from the surrounds greater than $500 \frac{1}{\mathrm{smm}}$.

Seeding was performed with a PIVtech generator using DiEthyl-Hexyl-Sebacat (DEHS) air soluble particles of $0.2-0.3 \mu \mathrm{m}$ typical diameter. This gives a Stokes number of approximately $210^{5}$, indicating the particle size is sufficiently low to follow all flow streamlines accurately [28].

Scattered laser light was captured by a monochrome cooled CCD pco.1600 camera with 1 GB of RAM. Images were digitised at 14 bits, with a resolution of $1600 \times 1200$ pixels. The camera was fitted with a $120 \mathrm{~mm}$ lens. The CCD size on the camera was $12.5 \mathrm{~mm}$ wide $9.38 \mathrm{~mm}$ high, giving a field of view at the most downstream plane of approximately $100133 \mathrm{~mm}$.

Image analysis was performed with PIVView software. Multi grid interpolation was used, starting at a coarse grid size of $128 \mathrm{px} 128 \mathrm{px}$ windows and finishing with refinement to $32 \mathrm{px} 32 \mathrm{px}$ over 3 passes. Standard FFT correlation was used, with two repeated correlations on $16 \mathrm{px}$ offset grids being performed resulting in minimal in-plane loss of pairs. Subpixel shifting was enabled on all passes with b-spline interpolation and peak detection by a Gaussian least squares fit from 3 points. The final grid size was 9974 nodes.

Calibration of the camera was performed using a grid that was photographed at all analysis plane locations, compensating for the increase in plane size due to perspective. The plane was located using the laser sheet, and then photographed to give an accurate scale.

\subsection{Sources of error}

Sampling error for averaged results was determined to be $3.7 \%$ in circulation and $0.0035 \mathrm{C}$ in location for the 400 total shots taken against a multiple representative sample of 2000 image pairs. Due to the nature of the manual focussing system there were induced errors, with differences in focus able to produce up to $0.04 \mathrm{C}$ error in core location. By implementing a particle pixel size threshold of no more than $2 \mathrm{px}$ at a brightness level of $4.5 \%$ of the total dynamic range, this error was reduced to $0.0015 \mathrm{C}$ in core location. Total error due to the calibration plane procedure was found to be a maximum of $0.18 \%$ in location and $0.22 \%$ in scale, due to minute differences in lateral calibration plane location. Seeding levels in the room were convergence tested such that the error from the seeding were not discernible from the randomness induced by the other errors. Spatial convergence was ensured by evaluating the $0.2 \mathrm{C}$ offset case at half the interrogation window size, effectively doubling the spatial resolution. This yielded errors of $\pm 2.7 \%$ in core radius and $\pm 0.0026 \mathrm{C}$ in location across the averaged sample size for the zoomed out condition used. As previously discussed, camera vibration was not observed at an appreciable level, with a maximum image migration of $0.06 \%$ measured over the course of an imaging run. The particle size was measured at an average of $1.5 \mathrm{px}$, giving an uncertainty in position of $0.03 \mathrm{px}$ [27]. Quantization errors were negligible due to 14 bit quantization. Any biases inherent in each run were minimised by having the each set of 400 images taken with one forward run of 200 images (plane moving from X17 to X11.5) and one backward run in the opposite direction; this way any errors in seeding or focus would be minimised. The total error in core location was found to be $\pm 0.008 \mathrm{C}$.

\section{Results and discussion}

Vortex radii can vary by up to $35 \%$ from instantaneous results if time averaged results are used due to vortex meandering and local fluctuations in velocity [22]. In addition to this, the velocity field will be smoothed, resulting in significant deviations in circulation and core size if time averaged results are used. However, it is still desired to have average values for core location, size and strength, and as such the results were analysed by a script based evaluation of each individual pair of images. These images were sequentially analysed in Matlab, with peak noise filtered by vorticity gradient as previously mentioned. To eliminate the influence of vortex shedding and low level noise on the calculation of tip vortex properties, all vorticity constructs except the tip vortex were filtered out. This was performed by computing contours at $10 \%$ of the peak vorticity and calculating the area enclosed by each individual structure. All structures except the largest were then eliminated, leaving only the tip vortex. The positive and negative vortices were evaluated separately, giving the positive and negative circulation magnitudes, location of the positive and negative vortex cores, and core radii. These data points were then combined and analysed for average values and variances. This allowed for an accurate calculation of instantaneous core size, as well as time-averaged values that could be used to represent the core characteristics and allow comparison between cases.

\subsection{Core paths}

The vortex centre within a plane is defined as the integral of the vorticity multiplied by the displacement, divided by the circulation [9]. This can be seen in Eqs. (1) and (2).

$X_{c}=\frac{1}{\Gamma} \int X \omega d S$

$Y_{c}=\frac{1}{\Gamma} \int Y \omega d S$

While this does not always align with the location of zero inplane velocity, it allows for consistent prediction of the centre of circulation intensity even when the vortex pair is migrating with an in plane motion, which would otherwise skew the core location 
significantly. It is also more robust than simply using the value of peak vorticity, as it is not significantly skewed by asymmetrical vortices or vorticity peaks in the result. As previously mentioned, these values were calculated at all image pairs, then averaged in Matlab. An example of the averaging is given below in Fig. 3. From this data, the core paths can be compared between cases.

Inspecting a selection of paths from across the cases investigated, as seen in Fig. 4, a basic migration trend emerges. At the far ends of the range ( $0.6 \mathrm{C}$ and $0.5 \mathrm{C}$ ) the migration is near linear, and predominantly vertical. At the negative end of the spectrum, the paths move upwards, while at the positive end they move downwards, similar to the theoretical predictions of Lewecke et al. [9]. This is due to the shear between the pair being minimal due to complimentary rotation, while at the periphery of the pairs there is no such rotation. This causes a shear between the vortex pair and the freestream flow, resulting in the migration of the vortex pair in the opposite direction to the outer velocity of the vortices, as can be seen in Fig. 5. At closer offsets, the motion is less vertically dominated, and takes on a more significant lateral component, as well as a significant rotational motion between the vortex pairs. As the configuration transitions between predominantly vertical motion to predominantly lateral motion, the magnitude of the migration increases significantly, as can be seen by the $80.5 \%$ difference between the 0.5 and 0.2 case. This is followed by a significant drop of $27.2 \%$ in the total migration between the 0.2 and

0.05 cases as the vortices interact more closely. The same effects can be seen on the negative side as it approaches the point of interaction, from 0.5 to 0.25 .

The positive offset case vortex paths are shown in Fig. 6. At the maximum offset $(0.5 \mathrm{C})$, the vortex pairs have little interaction, with minimal deviation in their paths. The separation between the vortex pair alters approximately linearly in the same amount as the variation in offset between the vanes. For this range of offsets the vortex pair separation does not significantly vary from the start to end of the domain, with the spacing increasing by an average of $0.024 \mathrm{C}$. The progressive increase in vortex pair migration as the vortices are brought together can also be seen in this figure, with a progressive increase in vertical migration from the $0.5 \mathrm{C}$ to $0.1 \mathrm{C}$ cases of $0.19 \mathrm{C}(101 \%)$.

At the $0.1 \mathrm{C}$ case, a rotation of the vortex pair has become evident, with significant curvature apparent to both the upstream and downstream vortex paths. This curvature occurs as a result of a differential in vortex strengths in the pair. As the circulation is higher on the downstream vortex, the weaker vortex is drawn into a rotational path around it. This results in a direction of rotation in the direction of the stronger vortex, despite the fact that its downwards shear is higher than that of the weaker vortex due to its increased circulation. Consequently, the path of the weaker (upstream vortex) is significantly longer than the stronger vortex, with a total migration of $0.660 \mathrm{C}$ as opposed to $0.522 \mathrm{C}$ for the downstream vortex. This can only occur when a combination of conditions are met, both the vortex proximity being sufficiently close to produce significant interactions of the high vorticity core regions, and the differential in strengths between the vortices being sufficient to promote rotation. With both cores having an average $R_{0.1}$ of $0.146 \mathrm{C}$ and the vortex separation distance between the cores being $0.274 \mathrm{C}$, this would indicate that significant vortex interactions which affect the strength of the upstream vortex begin to occur at a vortex spacing approximately equivalent to $2 \times R_{0.1}$. This is the spacing where the two vortex radii would just be intersecting.

As the upstream vortex passes closer to the rear vane, the rotational and horizontal migration of the vortex pair significantly increases. This can be seen in Fig. 7. With no rear vane the upstream vane's vortex core was located at approximately $0.1 \mathrm{C}$. This means the upstream vortex would pass by the downstream vane without direct impingement in the $0.3 \mathrm{C}$ and $0.25 \mathrm{C}$ cases. However, as the offset is further reduced ( $0.15 \mathrm{C}$ and $0.1 \mathrm{C}$ ) the upstream vortex will impinge on the downstream vane. This causes a reduction in the path lengths of both vortices, and

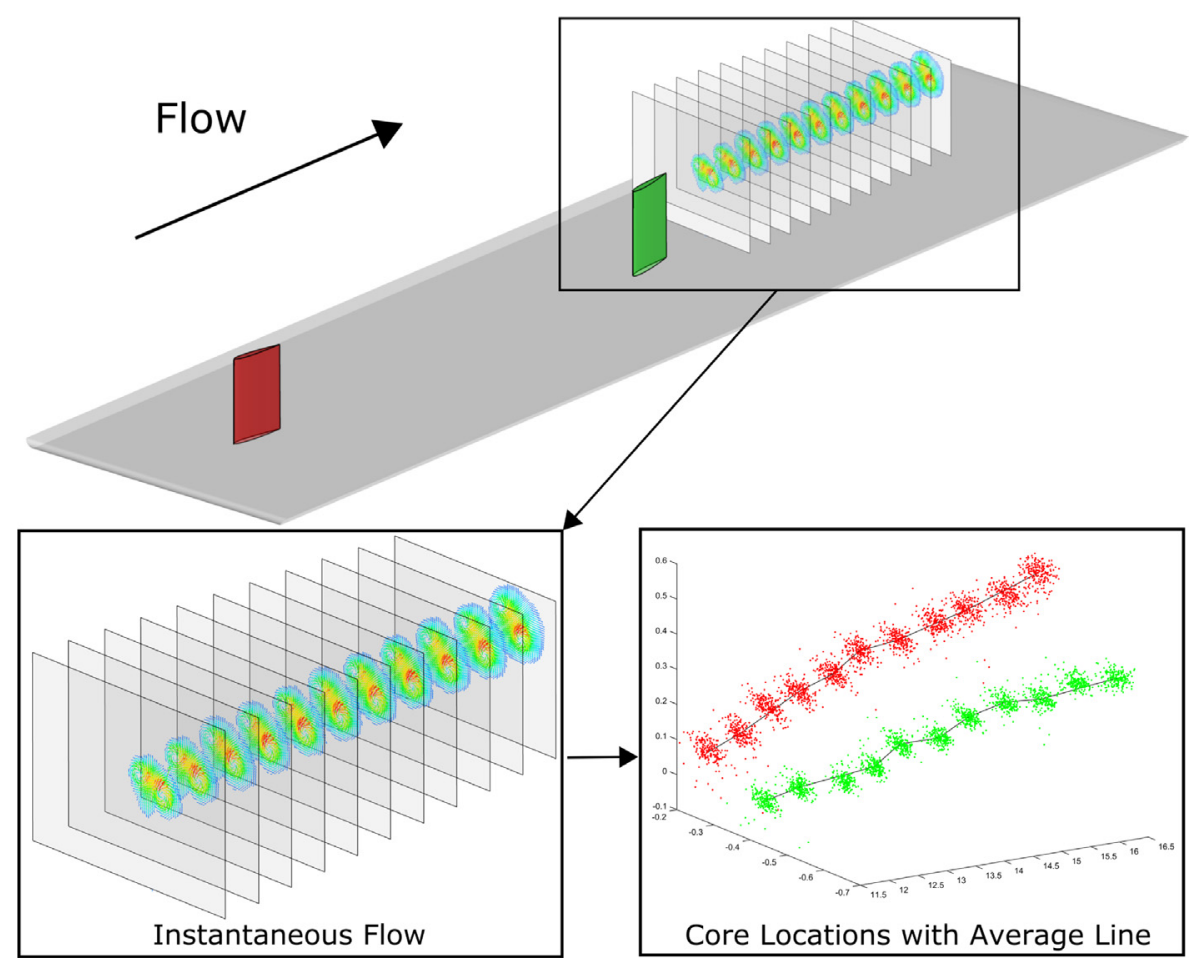

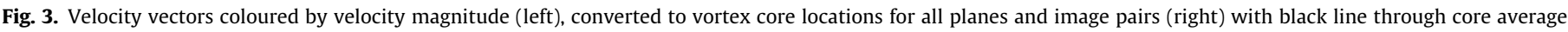
locations. 

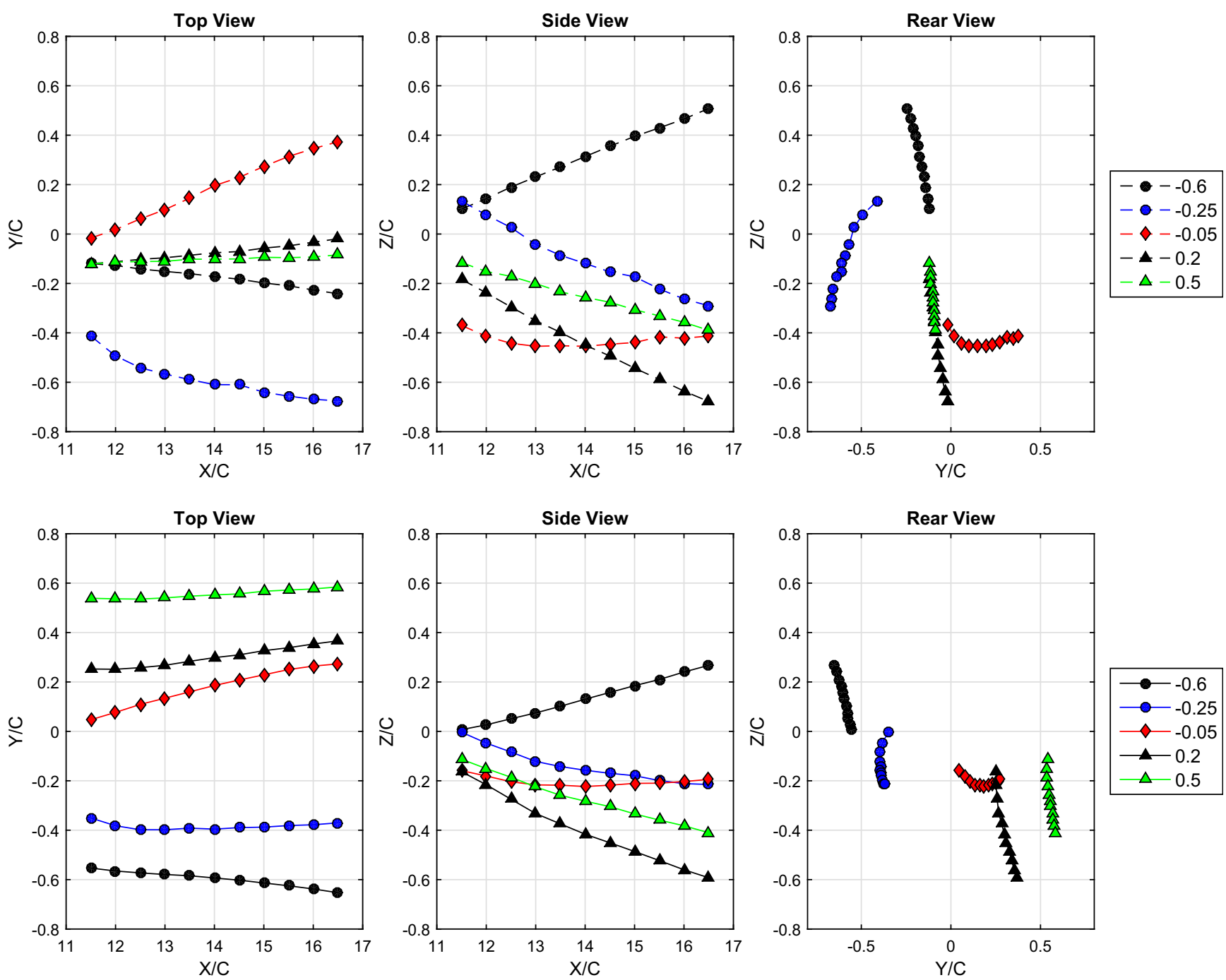

Fig. 4. Paths of upstream (dotted) and downstream (solid) vortices. Note the scale difference between the top and side views. Error in core location is $\pm 0.008 \mathrm{C}$.

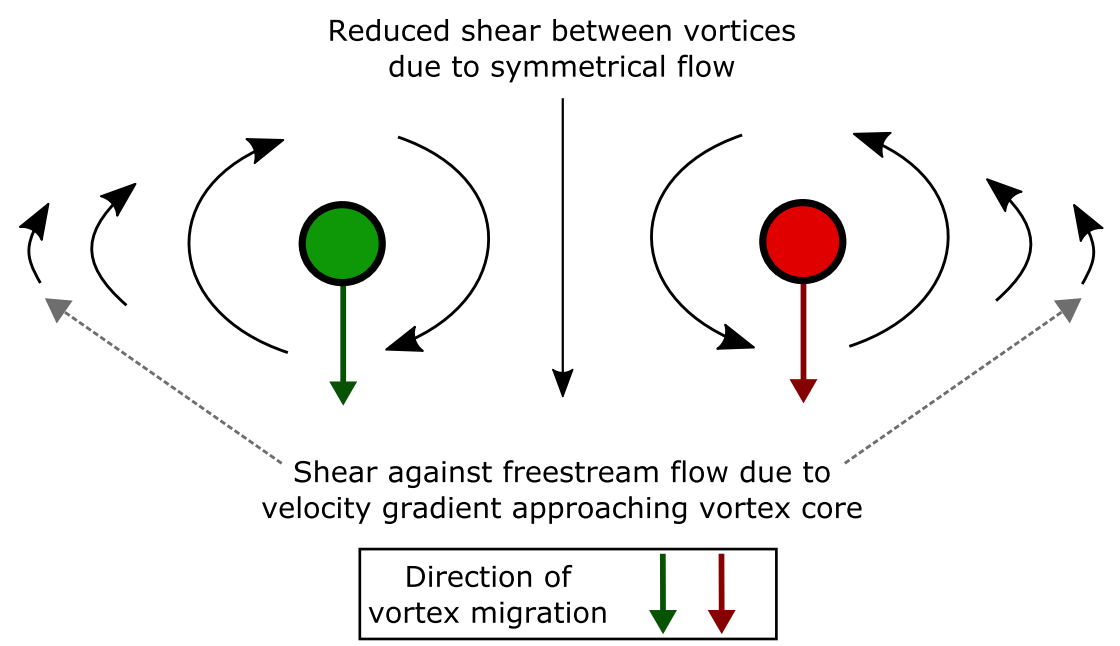

Fig. 5. Schematic of vortex core migrations for equal circulation counter-rotating cases.

increases the separations. At the $0.2 \mathrm{C}$ offset the $R_{0.1}$ of the upstream vortex marginally impinges on the suction surface of the downstream vane. This has caused a reduction in downstream path length from $0.216 \mathrm{C}$ to $0.128 \mathrm{C}$. As such, the interaction between the downstream and upstream vortices post vane must 

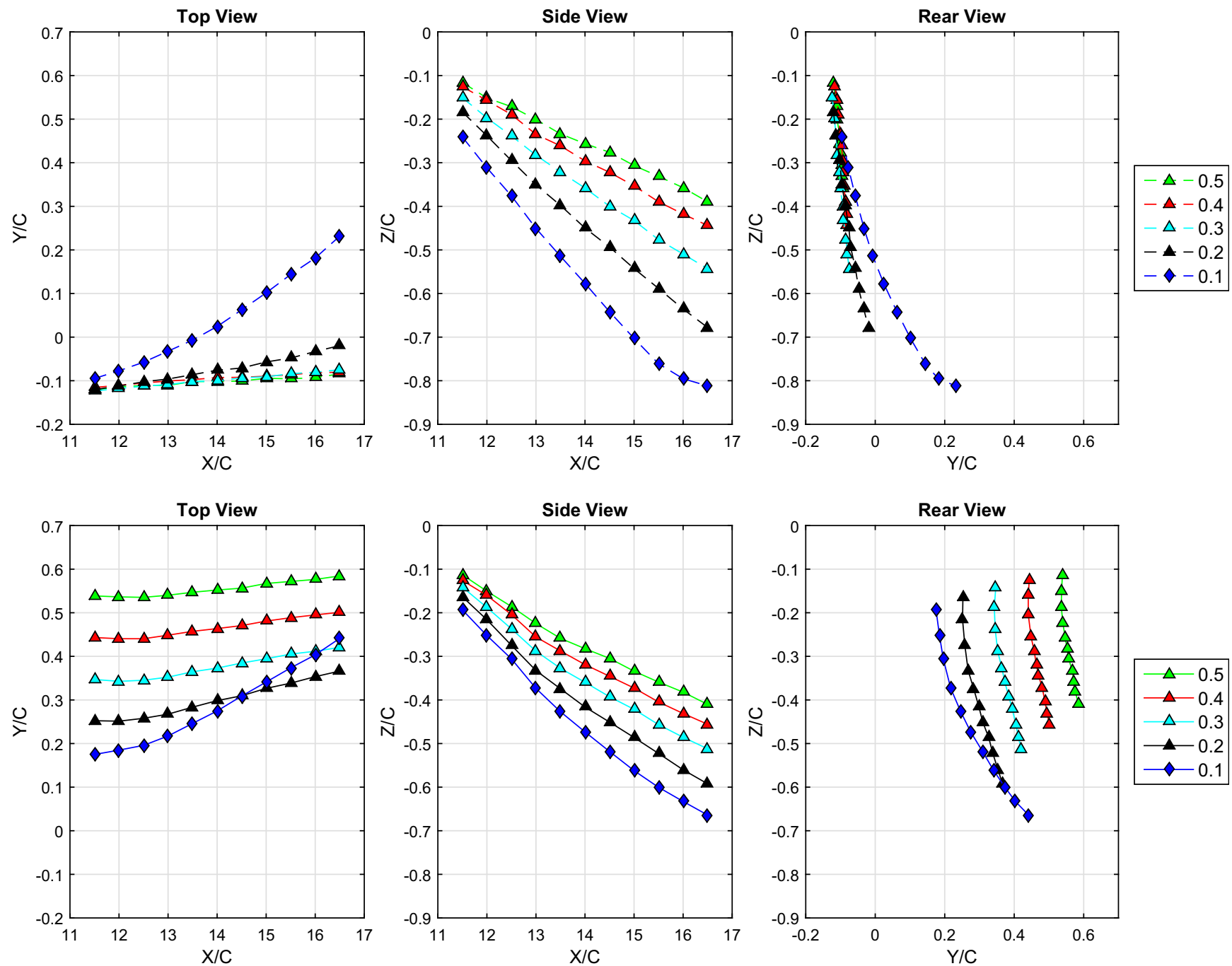

Fig. 6. Paths of upstream (dotted) and downstream (solid) vortices. Note the scale difference between the top and side views. Error in core location is $\pm 0.008 \mathrm{C}$.

be strongest at $0.25 \mathrm{C}$, while the point of impingement is located at $0.1 \mathrm{C}$.

The rate of rotation by which the two vortices orbit each other was calculated through a linear approximation of the change in angle of the line drawn between the two vortex cores. This can be seen diagrammatically in Fig. 8. By looking at these rotational rates in Fig. 9, it can be seen that the lowest angular core velocities are achieved at $0.1 \mathrm{C}$, the point where the upstream core would impact the quarter chord of the downstream vane if no deviations occurred as a result of the presence of the second vane. Rotational rate peaks occur at $0.2 \mathrm{C}$ and $\mathrm{OC}$, at peaks of 19.57 and 17.74 degrees/C respectively. The peaks are caused by a combination of high strength interaction and close vortex proximity. Of interest is the increased rotational rate of the $0.2 \mathrm{C}$ case compared to the stronger interacting $0.25 \mathrm{C}$ case. Closer inspection revealed that the $0.2 \mathrm{C}$ rotation was high at the start of the domain, however rapidly reduced after X14, while the $0.25 \mathrm{C}$ case remained near constant. As such, the partially impinged interaction of the

$0.2 \mathrm{C}$ offset causes a strong initial interaction as it affects the vortex formation. However, the $0.2 \mathrm{C}$ interaction causes a more rapid reduction of the vortex strengths as they progress downstream, with a subsequent reduction in rotational rate, while the $0.25 \mathrm{C}$ interaction shows far less reduction. Between $0.35 \mathrm{C}$ and $0 \mathrm{C}$ there are the most significant gradients of rotational rate due to the tran- sition of the upstream vortex location around the vane. On the negative side of this rotational peak the rotation rates trend towards the values seen on the far positive regions, as would be expected as the vortex separations become significant again.

The initial vortex separations between the vortex pairs remain relatively consistent through the range of near field interactions from $0.35 \mathrm{C}$ to $0.2 \mathrm{C}$, however dip slowly, and then drop to their lowest separation at $0.25 \mathrm{C}$. While the initial separations decrease towards the $0.25 \mathrm{C}$ offset, the final separations remain far more constant until $0.15 \mathrm{C}$ offset. This indicates that for a given vortex core size the vortices will attempt to reach an equilibrium separation distance, in this case approximately $1.6 R_{0.1}$. The initial core spacing in the $0.25 \mathrm{C}$ case is the smallest, at approximately 1 core radius. Bringing the vortices closer than this will begin to destroy the upstream vortex significantly. As the upstream vortex impinges on the vane it causes the vortices to increase both their initial and final separation distances, as can be seen in the points from $0.2 \mathrm{C}$ to $0.1 \mathrm{C}$. At the point of complete impingement the separation has become largest, and the rotation smallest, indicating that this is no longer a point of significant interaction, but rather the downstream vane has significantly reduced the strength of the upstream vortex during the direct vane/vortex interaction. This configuration also displays a smaller difference between the initial and final separations than the surrounding points on the 

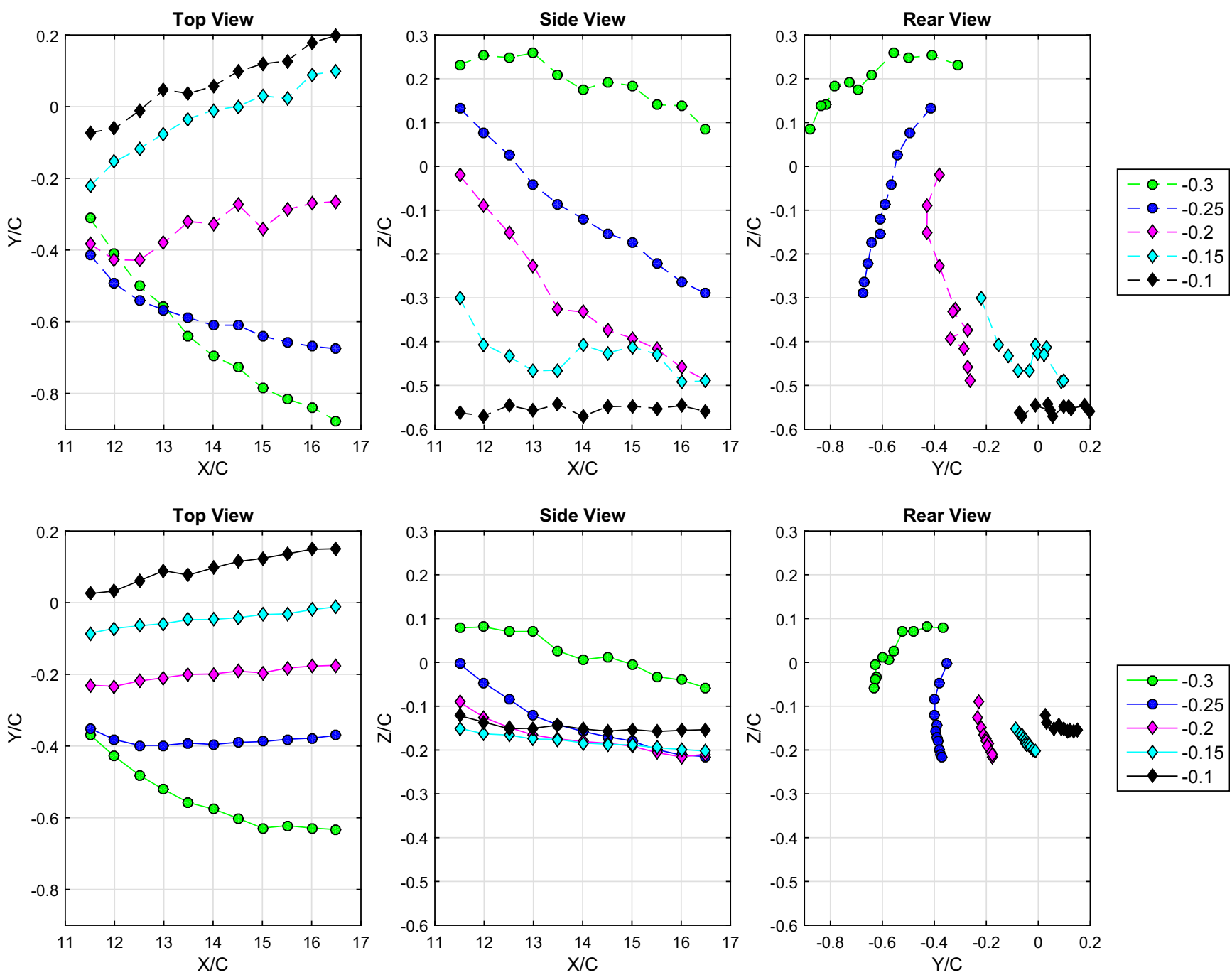

Fig. 7. Paths of upstream (dotted) and downstream (solid) vortices. Note the scale difference between the top and side views. Error in core location is $\pm 0.008 \mathrm{C}$.

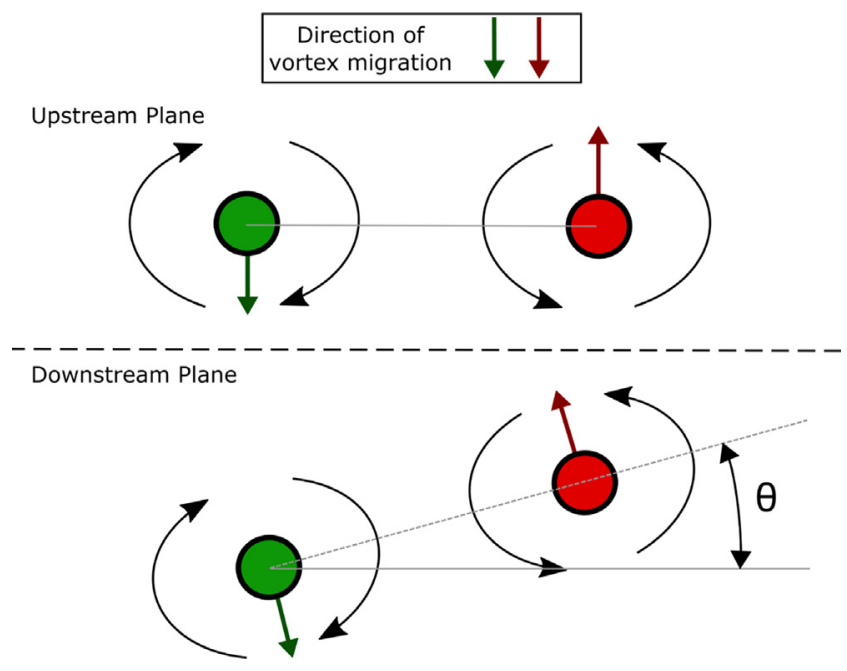

Fig. 8. Schematic of rotation angle calculation for vortex pairs.

negative side as the vortices have reached a steady equilibrium state in the flow and the subsequent interactions are weak.

\subsection{Core sizes}

While the vortices remain near a uniform Lamb-Oseen distribution at the far offsets, at nearer offsets significant partial straining occurs from the influence of the vortex interaction. This causes a skew in the shape of the vortex core that changes its primary axis as the vortex pair rotates downstream. This prevents the fitting of a Lamb-Oseen distribution of vorticity to the results. Consequently, to calculate the core radius, the area bounded by the isoline of $10 \%$ of the peak vorticity within the plane has been used in both the positive and negative circulations, as used by Manolesos [29]. While this area can vary significantly from a circle, an effective radius can be calculated from Eq. (3) by assuming approximate circularity.

$R_{0.1}=\sqrt{\frac{A_{0.1}}{\pi}}$

The removal of noise from the data via the previously mentioned filtering ensures that only the area of the core itself is processed, and not the surrounding flow features or noise outside the core. By comparing this method to a Lamb-Oseen approximation, it was found that the spatial sampling resolution could result in a $15 \%$ maximum error in peak vorticity. This translated to a $1.5 \%$ maximum error in the $10 \%$ peak vorticity, giving a maximum core 

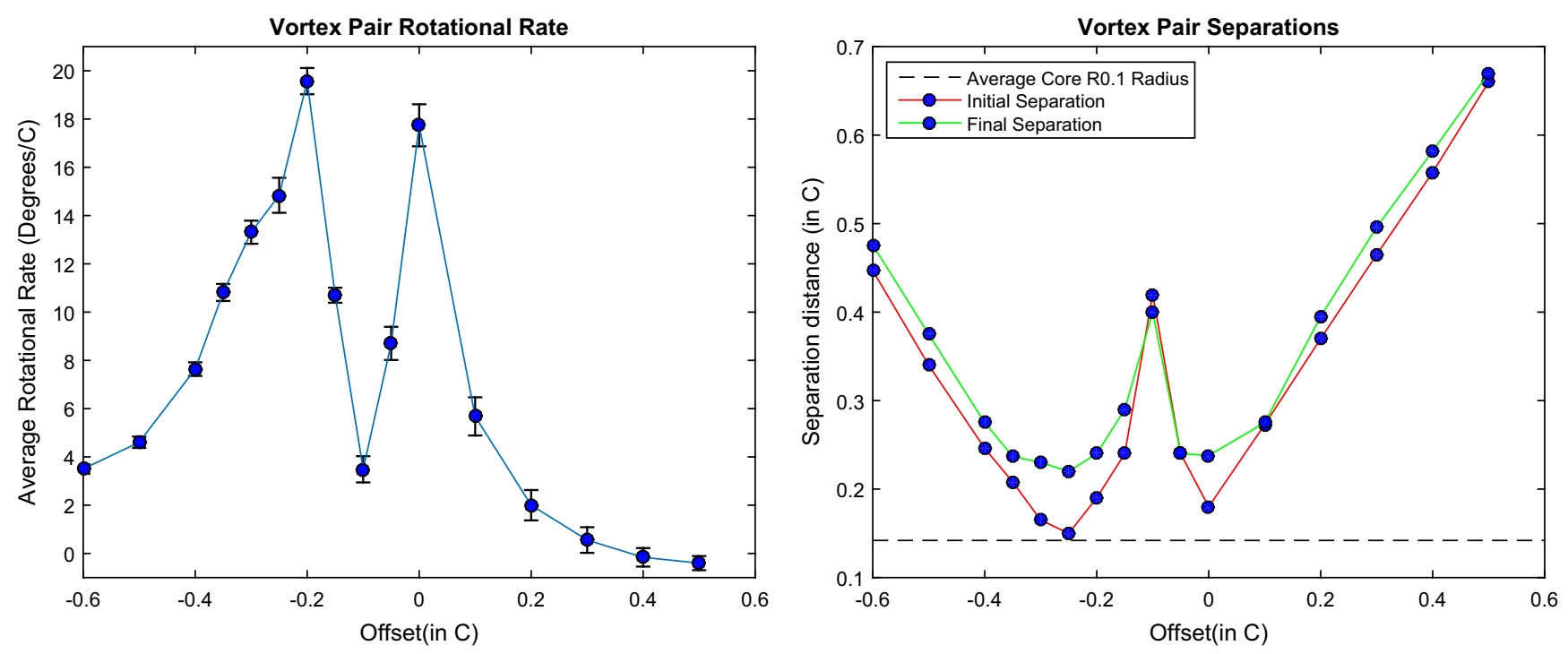

Fig. 9. Rotational rates of the vortex pairs (left) and average vortex separations (right). Error in vortex separation is $\pm 0.005 \mathrm{C}$.

radius error of $5 \%$ per image pair, which was considered acceptable for this analysis. This was confirmed by evaluating the $0.2 \mathrm{C}$ offset case at double the spatial resolution as previously mentioned, yielding errors of $\pm 2.7 \%$ in core radius across the averaged sample size.

Initial and final values for core radius were calculated by linearly approximating the gradients of core radius across the domain, reducing the effect of statistical variance on the measured sizes. These core radii can be seen below in Fig. 10.

At the $0.3 \mathrm{C}$ offset a significant reduction in initial core radius can be seen for the downstream vortex. However, as these progress through the domain the downstream vortex grows in size by $0.024 \mathrm{C}$, while the upstream vortex radius decreases by $0.025 \mathrm{C}$. This is the only near-field interaction case observed to have a significant trend of growth in the downstream vortex, and is also a local minima before the increase in initial downstream vortex size to the peak at $0.2 \mathrm{C}$ offset. Between $0.25 \mathrm{C}$ to $0.2 \mathrm{C}$, the previously identified peak of vortex interaction, there is a transition from a larger initial upstream radius to a larger initial downstream radius. While this change is small in magnitude, the final downstream vor- tex size peak at the $0.25 \mathrm{C}$ case has a more significant change, indicating that the strong interaction has resulted in the transfer of energy from the upstream vortex to the downstream vortex throughout the domain, causing an increase in the size of the downstream vorticity field.

As the interaction approaches the point of impingement, the final size of the upstream vortex decreases to a minima at

$0.15 \mathrm{C}$. As the upstream vortex moves closer to the tip, its strength is significantly reduced by the counter-acting vorticity, resulting in these decreases in core size. At the point of impingement $(0.1 \mathrm{C})$ there is a marked decrease in downstream vortex cores size. However, the upstream vortex size has increased by $17 \%$ at this point from the $0.15 \mathrm{C}$ case. The reason for this was not apparent from the results, however it is likely related to the downstream vortex stripping vorticity from the upstream vortex when slightly offset, while in the direct impingement case the downstream vortex itself is significantly weakened, and as such cannot draw energy from the upstream vortex as successfully. As the offset increases towards the positive side, there is a steady increase in the final core
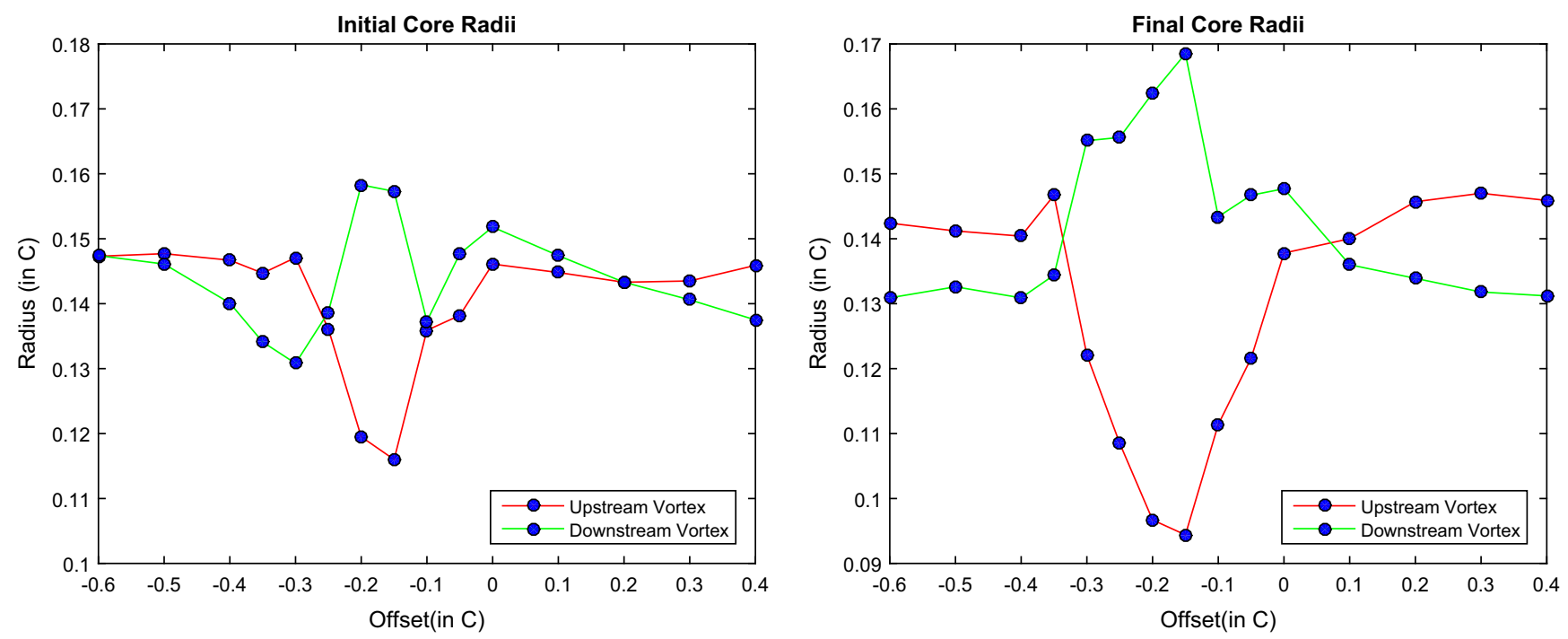

Fig. 10. Core radii for all cases at X11.5 (Initial, left) and X16.5 (Final, right). 
radii for both the upstream and negative vortices, with less clear trends in the initial size.

\subsection{Vortex meandering}

In addition to the circulation and core location changing as the vortices pass through the domain, they also vary with respect to time. Vortex meandering is the phenomenon of random vortex motions and oscillations that result from any turbulent vortex flow. While the origins of meandering are disagreed upon [7,30$32]$, it is still important to characterise, as it changes the predictability of the flowfield, particularly in real world scenarios. Given the large and effectively random sample of image pairs taken, the statistics of the variance of both circulation and core location can be used for analysis of the meandering magnitudes. While the period, frequencies and amplitudes of small oscillations cannot be evaluated with non-temporally resolved data, the total magnitudes of displacements and the location distribution of the meandering can be determined with non temporally resolved data and a sufficiently large sample size. Such methodology has been used by Miller et al. [33] and Rokhsaz [34] at $30 \mathrm{~Hz}$, as well as Heyes et al. [35] at $5 \mathrm{~Hz}$. The core variance was calculated as the standard deviation of the radial distance from the average core location, while the circulation variance was calculated from the standard deviation of the difference from instantaneous circulation to average circulation, divided by the average circulation on the plane. The division by the average circulation was used to remove bias caused by low circulation cases and planes, as this would lead to low circulation cases seemingly having less fluctuation magnitude.

Inspecting the core variances in Fig. 11, it can be seen that the natural tendency of the cores in the far interacting cases is to maintain a near constant meandering magnitude throughout the domain investigated. From the $0.2 \mathrm{C}$ to $0.4 \mathrm{C}$ cases it can be seen that the end variance is less than the start variance for the downstream vortices, and very similar for the upstream vortices, showing that the initial meandering motion is be caused by the formation of the vortices. The shear layers shed off the vanes may provide the initial perturbations, resulting in the fluctuating deviation of the core location. As the flow travels further downstream, these spanwise vortices will be dampened out by viscous effects, as well as flow entrainment into the streamwise vortices. These vortices are too far apart for the Crow instability to have a

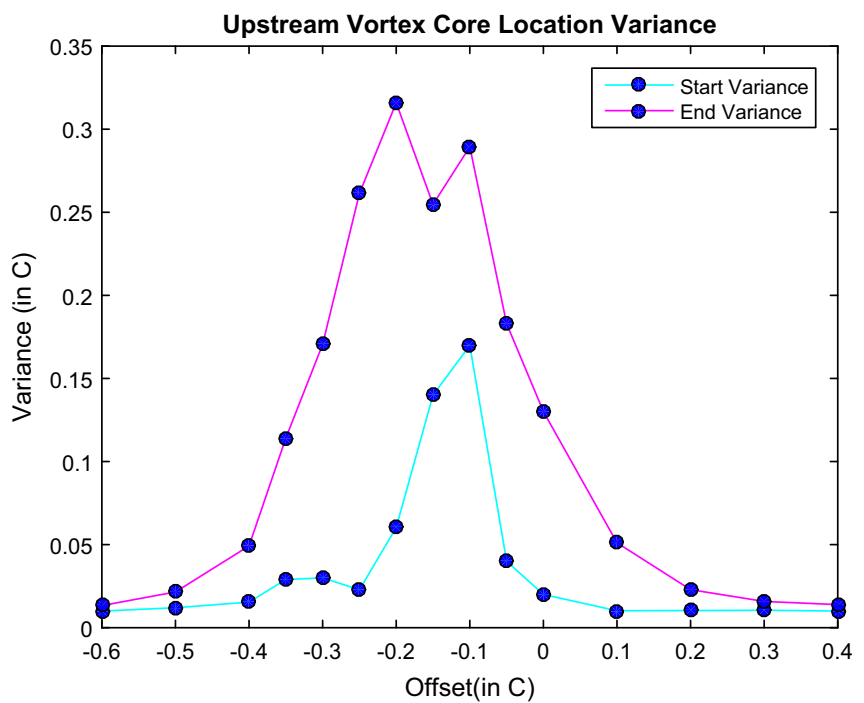

significant effect within this domain. This explains the reduction of the meandering magnitudes as the vortices progress.

As the interactions of the vortices become stronger, their meandering magnitudes significantly increase. Between $0.2 \mathrm{C}$ and

$0.05 \mathrm{C}$ the start variance of the upstream vortex significantly increases. This is in the region of the upstream vortex $R_{0.1}$ intersecting the suction side of the downstream vane. At $0.1 \mathrm{C}$ offset there is a peak variance of $0.17 \mathrm{C}$, which is greater than $R_{0.1}$. This indicates that in near field interactions the upstream vortex is fluctuating from one side of the vane to the other, creating a large spread of core locations. This increase is co-incident with the reduction in vortex pair rotation angle between $0.2 \mathrm{C}$ and $\mathrm{OC}$. The downstream vortex is far less affected by these variations, with a maximum increase in start variance of $0.0196 \mathrm{C}$ over the case with the least variance.

While the start variance is proportional to the proximity of the incident vortex to the downstream vane, the end variance is more dependent on the magnitude of the interaction. This is particularly true for the downstream vortex, which achieves a variance peak of $0.155 \mathrm{C}$ at $0.3 \mathrm{C}$ offset and a significant increase in meandering from $0.35 \mathrm{C}$ to $0.15 \mathrm{C}$. This is accompanied with a wider spread of meandering in the upstream case, with significant increases in meandering once the vortex separation drops below $0.275 \mathrm{C}$ ($0.4 \mathrm{C}$ and $0.1 \mathrm{C}$ offsets). These downstream vortex proximities are sufficiently close to allow for instabilities to be formed between the vortices, creating the meandering observed. In both vortices, the peak in variance at the downstream end of the domain occurs at a more negative offset than either vortices start peak. This indicates that the low pressure region on the suction side of the downstream vane and resultant adverse pressure gradient is enhancing the instabilities of the vortex pair further downstream.

Further investigation of the nature of the meandering shows a clear instability in the upstream vortex, as can be seen in Fig. 12. At larger offsets $(0.3 \mathrm{C}$ in figure) the presence of any sinusoidal deviation is minimal, with only a slight skew observed in the upstream vortex. As the offset is brought closer $(0.1 \mathrm{C})$ a clear deviation of points at approximately 45 degrees to the line between the vortex centres can be seen. This is indicative of a sinusoidal deviation, similar to the uneven Crow instability previously identified in computational work by the authors [11]. The deviation is far more prominent for the upstream vortex than the downstream vortex, which has an approximately circular distribution of locations. The reason for this inconsistency was not apparent from the

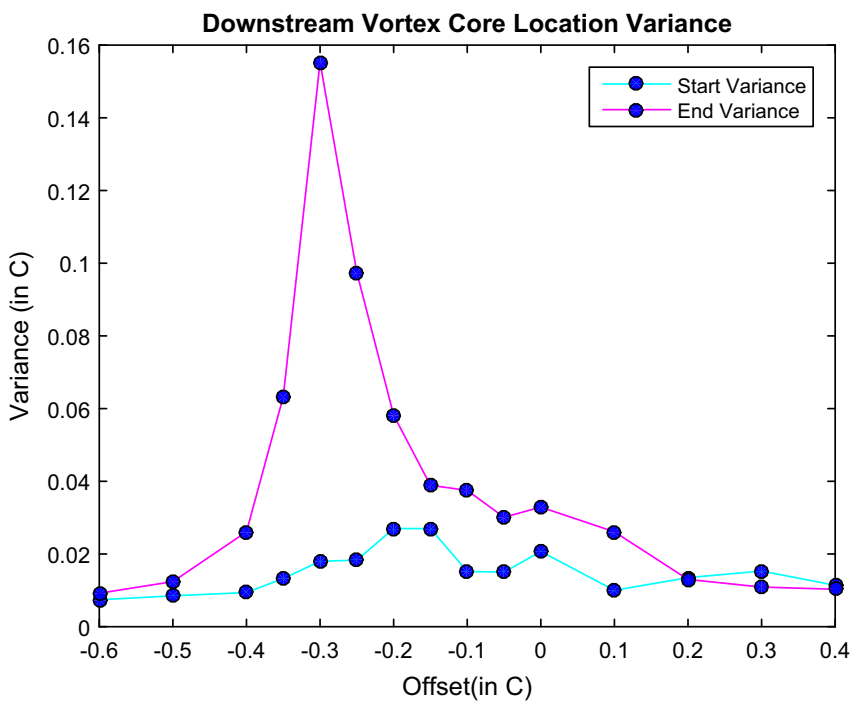

Fig. 11. Core location standard deviation of upstream vortex (left) and downstream vortex (right). Note the scale difference between the two plots. 

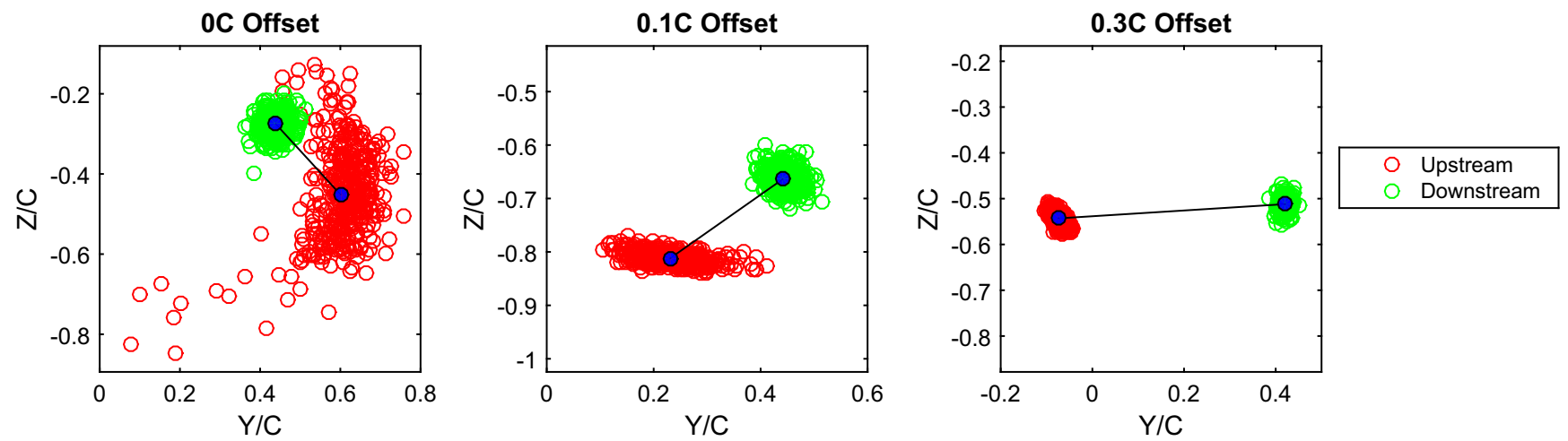

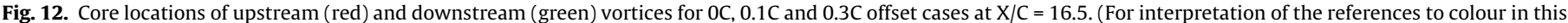
figure legend, the reader is referred to the web version of this article.)

results, however it is likely due to the longer path of the upstream vortex, in addition to reduced vortex strength from the initial vane/ vortex interaction. As the offset is further reduced, the upstream vortex is drawn into the velocity field of the downstream vortex, resulting in a curvature of its sinusoidal deviations. This can be seen in the $0 \mathrm{C}$ offset of Fig. 12. The same trends were seen when approaching the vortex impingement from negative offsets.

The variances in circulation followed similar trends to that of the core location, so are not presented here. The consistency in these trends indicates that the damping mechanisms which smoothen out the location meandering in the far offset cases also calm the fluctuations of the vortex strength. As the increased swirl velocities of high circulation will be reduced more rapidly by shear than the lower velocities associated with low circulation, it is expected that these fluctuations would be reduced as the vortices pass through the flowfield, as long as there is not a significant instability present. Of more interest is the increase in circulation variance near the points of higher interaction. In the near field, the normalised circulation variances were increased by 0.078 (75\%) and 0.428 (471\%) for the downstream and upstream cases respectively. In the far field, these variances were increased by $0.20(171 \%)$ and 0.4551 (932\%) for the downstream and upstream cases respectively. This indicates that the close interactions are influential in the magnitude of the circulation fluctuations well downstream from the initial interaction of the vortex with the vane. As such, the interactions of the vortices with one another can be observed to destabilise the cores and enhance the energy transfer between the vortices.

\section{Conclusion}

Wind tunnel experimentation has been performed to characterise the behaviour of the downstream interactions of the vortex pair produced by two offset vanes, spaced $10 \mathrm{C}$ apart in the streamwise direction. 1.5 aspect ratio NACA0012 wings at 8 degrees angle of attack and a Reynolds number of 70000 were used for this study. Several lateral offsets were used to examine the effects of vortex proximity on the resulting vortex sizes and paths.

For far positive offset cases, the vortex pair migrated downwards, while for far negative offsets the pair migrated upwards. No vortex rebound was observed within the domain, indicating the vanes were sufficiently high above the floor to be free of ground effect. At close offset cases, the motions of the vortex pairs shifted from predominantly vertical to predominantly lateral, with increased rotation of the pairs. The rotational rate of the vortex pair had two peaks at $0.20 \mathrm{C}$ offset and $0 \mathrm{C}$, with a minima at $0.1 \mathrm{C}$, the point of core impingement. This is consistent with the location of the core with no downstream vane present. At this point the size and strength of both vortices has been significantly reduced as a result of the destructive interference in the formation stage of the downstream vortex. This is responsible for the low rotational rate. $0.25 \mathrm{C}$ produced the strongest interactions, with the second highest rotational rate and highest vortex size changes, combined with closest vortex pair proximity. The separation between the vortices in this condition was approximately $R_{01}$. This indicated that placing a vortex one core radius from the suction side of a vane is preferable for maximum interaction strength, while impacting the vortex on the quarter chord causes the most significant vortex destruction.

The vortex meandering was found to be dependent on the proximity of the interaction, with closer proximities producing higher meandering levels. The strength of the shear layer shedding and instabilities introduced by the unequal strength interaction were found to be significant factors. The meandering magnitudes were found to be more closely related to the strength of the interaction than the destruction of the vortices, with the $0.25 \mathrm{C}$ case having the largest meandering magnitude and steady decreases on either side of this. Downstream vortex meandering was found to be more sensitive to the strength of interaction than the upstream vortex, with a typically lower meandering growth at further offset cases. Near offset cases produced a clearly observable instability in the upstream vortex only, with the 45 degree deviations being drawn around the stronger vortex in a curved manner as the separation distance was reduced. Circulation fluctuations followed similar trends, demonstrating a link between circulation and core location in meandering.

The rich dynamics observed and large changes in vortex state resulting from small offset changes near the point of impingement indicate that the traditional method of exploring only 3 or 4 offsets may not be sufficient when predicting the paths of a counter rotating pair produced in this manner. The presence of vortex meandering over longer distances would further amplify this problem, as the transient changes in location of the initial vortex prior to interaction with the downstream structure will result in large changes of the resultant pair's location and size. As such, in systems where consistent vortex behaviour is required, the counter-rotating pair should be spaced at as high an offset as feasible.

\section{References}

[1] K.J. Forster, T.R. White, Numerical investigation into vortex generators on heavily cambered wings, AIAA J. 52 (5) (2014) 1059-1071, http://dx.doi.org/ 10.2514/1.J052529. <http://arc.aiaa.org/doi/abs/10.2514/1.J052529>.

[2] K.J. Forster, S. Diasinos, T.J. Barber, G. Doig, Computational analysis of backwards facing vortex generators for boundary layer mixing applications, in: 19th Australasian Fluid Mechanics Conference (December), 2014, p. 341. 
[3] L.A.A. Pereira, M.H. Hirata, N.M. Filho, Wake and aerodynamics loads in multiple bodies-application to turbomachinery blade rows, J. Wind Eng. Ind. Aerodyn. 92 (2004) 477-491, http://dx.doi.org/10.1016/j.jweia.2004.02.001.

[4] M. Toloui, L.P. Chamorro, J. Hong, Detection of tip-vortex signatures behind a 2.5 MW wind turbine, J. Wind Eng. Ind. Aerodyn. 143 (2015) 105-112, http:// dx.doi.org/10.1016/j.jweia.2015.05.001.

[5] D. Hummel, Formation flight as an energy-saving mechanism, Israel J. Zool. 41 (3) (1995) 261-278.

[6] C.M. Velte, M.O. Hansen, V.L. Okulov, Multiple vortex structures in the wake of a rectangular winglet in ground effect, Exp. Thermal Fluid Sci. 72 (2016) 31-39, http://dx.doi.org/10.1016/j.expthermflusci.2015.10.026. < ittp:// www.sciencedirect.com/science/article/pii/S0894177715003027>.

[7] D. Garmann, M. Visbal, Interactions of a streamwise-oriented vortex with a finite wing, J. Fluid Mech. 767 (2015) 782-810, http://dx.doi.org/10.1017/ jfm.2015.51. S0022112015000518>

[8] S. Crow, Stability theory for a pair of trailing vortices, 1970. doi:http://dx.doi. org/10.2514/3.6083. <http://arc.aiaa.org/doi/abs/10.2514/3.6083>.

[9] T. Leweke, S. Le Dizès, C.H.K. Williamson, Dynamics and instabilities of vortex pairs, Annu. Rev. Fluid Mech. 48 (2016) 507-541, http://dx.doi.org/10.1146/ annurev-fluid-122414-034558.

[10] R.E. Gordnier, M.R. Visbal, Numerical simulation of the impingement of a streamwise vortex on a plate, Int. J. Comput. Fluid Dynam. 12 (1) (1999) 4966.

[11] K.J. Forster, T. Barber, S. Diasinos, G. Doig, Numerical investigation of streamwise vortex interaction, SAE Technical Paper.

[12] R. Klein, Simplified equations for the interaction of nearly parallel vortex filaments, J. Fluid Mech. 288 (1995) 201-248. <http://journals.cambridge.org/ abstract_S0022112095001121>.

[13] D. Fabre, L. Jacquin, A. Loof, Optimal perturbations in a four-vortex aircraft wake in counter-rotating configuration, J. Fluid Mech. 451 (2002) 319-328, http://dx.doi.org/10.1017/S0022112001006954.

[14] S.E. Widnall, The structure and dynamics of vortex filaments, Annu. Rev. Fluid Mech. 7 (1975) 141-165, http://dx.doi.org/10.1146/annurev. fl.07.010175.001041.

[15] P. Chatelain, A. Curioni, M. Bergdorf, D. Rossinelli, W. Andreoni, P. Koumoutsakos, Billion vortex particle direct numerical simulations of aircraft wakes, Comput. Methods Appl. Mech. Eng. 197 (2008) 1296-1304, http://dx.doi.org/10.1016/j.cma.2007.11.016.

[16] C.-Y. Tsai, S.E. Widnall, The stability of short waves on a straight vortex filament in a weak externally imposed strain field, J. Fluid Mech. 73 (1976) 721, http://dx.doi.org/10.1017/S0022112076001584.

[17] K. Rokhsaz, R. Rebours, S.R. Foster, Quantitative measurements of wake vortex motion in a water tunnel, in: 39 Aerospace Sciences Meeting and Exhibit, no. January, 2001. doi:http://dx.doi.org/10.2514/6.2001-111.

[18] A. Inasawa, F. Mori, M. Asai, Detailed observations of interactions of Wingtip vortices in close-formation flight, J. Aircraft 49 (1) (2012) 206-213, http://dx. doi.org/10.2514/1.C031480. < <http://arc.aiaa.org/doi/abs/10.2514/1. C031480>.

[19] S. Pasche, F. Gallaire, M. Dreyer, M. Farhat, Obstacle-induced spiral vortex breakdown, Exp. Fluids 55 (8) (2014) 1784, http://dx.doi.org/10.1007/s00348014-1784-7. <http://link.springer.com/10.1007/s00348-014-1784-7>.
[20] M. Hall, A new approach to vortex breakdown, Proc. Heat Transfer Fluid Mech. Inst. (1967) 319-340.

[21] J.J. Cassidy, H.T. Falvey, Observations of unsteady flow arising after vortex breakdown, J. Fluid Mech. 41 (04) (1970) 727, http://dx.doi.org/10.1017 S0022112070000873. S0022112070000873>.

[22] A.F.K. Yeung, B.H.K. Lee, Particle image velocimetry study of wing-tip vortices J. Aircraft 36 (2) (1999) 482-484, http://dx.doi.org/10.2514/2.2460.

[23] T. Barber, P. Kurts, Downstream evolution of wingtip vortices produced from an inverted wing, Aeronaut. J. 119 (January) (2015) 747-763, http://dx.doi.org/ 10.1017/S0001924000010800. <http://www.journals.cambridge.org/ abstract S0001924000010800>.

[24] R.F. Huang, C.L. Lin, Vortex shedding and shear-layer instability of wing at lowReynolds numbers, AIAA J. 33 (8) (1995) 1398-1403, http://dx.doi.org/ $10.2514 / 3.12561$.

[25] J.-H. Yoon, S.-J. Lee, Direct comparison of 2D PIV and stereoscopic PIV measurements, Meas. Sci. Technol. 13 (2002) 1631-1642, http://dx.doi.org/ $10.1088 / 0957-0233 / 13 / 10 / 317$.

[26] C.D. Saunter, Quantifying subpixel accuracy: an experimental method for measuring accuracy in image-correlation-based, single-particle tracking, Biophys. J . 98 (8) (2010) 1566-1570, http://dx.doi.org/10.1016/j. bpj.2009.12.4297.

[27] C.W.M. Raffel, J. Kompenhas, Particle Image Velocimetry, A Practical Guide Springer, Berlin, Germany, 1998.

[28] C.A. Cruz, Experimental and numerical characterization of turbulent slot film cooling, ProQuest, 2008.

[29] M. Manolesos, S.G. Voutsinas, Experimental investigation of the flow past passive vortex generators on an airfoil experiencing three-dimensional separation, J. Wind Eng. Ind. Aerodyn. 142 (2015) 130-148, http://dx.doi. org/10.1016/j.jweia.2015.03.020.

[30] L. Jacquin, D. Fabre, P. Geffroy, E. Coustols, The properties of a transport aircraft extended near field: an experimental study, in: AIAA conference proceedings, vol. 1038, 2001

[31] S.J. Beresh, J.F. Henfling, R.W. Spillers, Meander of a fin trailing vortex measured using particle image velocimetry, in: 47th AIAA Aerospace Sciences Meeting Including The New Horizons Forum and Aerospace Exposition, no. January, 2009.

[32] K. Rokhsaz, T.S. Miller, Theoretical and experimental investigation of the selfinduced oscillations of a single vortex filament, in: 32nd AIAA Fluid Dynamics Conference and Exhibit, no. June, 2002. doi:http://dx.doi.org/10.2514/6.20023304

[33] T.S. Miller, L.K. Kliment, K. Rokhsaz, Analytical investigation of co-rotating vortex filaments with experimental verification, in: 33rd AIAA Fluid Dynamics COnference and Exhibit, no. June, 2003. doi:http://dx.doi.org/10.2514/6. 20033601.

[34] K. Rokhsaz, L.K. Kliment, Experimental investigation of co-rotating vortex filaments in a water tunnel, in: 32nd AIAA FLuid Dynamics Conference and Exhibit, no. June, 2002. doi:http://dx.doi.org/10.2514/6.2002-3303.

[35] A. L. Heyes, R.F. Jones, D.A. Smith, Wandering of wing-tip vortices, in: 12th International Symposium on Applications of Laser Techniques to Fluid Mechanics, 2004, p. 35-3. 